\title{
Effects of Different Storage on Qualities of Adzuki and Red Kidney Beans
}

\author{
Shan $\mathrm{Wu}^{1,2}$, Shuo Feng ${ }^{1,2} \&$ Michiyuki Kojima ${ }^{1}$ \\ ${ }^{1}$ Department of Food Production Science, Obihiro University of Agriculture and Veterinary Medicine, Obihiro, \\ Hokkaido, Japan \\ ${ }^{2}$ United Graduate School of Agricultural Sciences, Iwate University, Morioka, Iwate, Japan \\ Correspondence: Michiyuki Kojima, Department of Food Production Science, Obihiro University of Agriculture \\ and Veterinary Medicine, Obihiro, Hokkaido, Japan. Tel: 81-015-549-5547. Fax: 81-015-549-5547. E-mail: \\ kojima@obihiro.ac.jp
}

Received: February 3, 2015 Accepted: March 11, 2015 Online Published: March 20, 2015

doi:10.5539/jfr.v4n3p103 URL: http://dx.doi.org/10.5539/jfr.v4n3p103

\begin{abstract}
This is the first study to report the effects of frozen soil storage on adzuki and red kidney beans. Adzuki and red kidney beans were stored for 15 months in polyethylene or paper bags under three different conditions: natural cold energy storage in frozen soil, storage in a freezer at $-20{ }^{\circ} \mathrm{C}$, and storage at a constant temperature of $25{ }^{\circ} \mathrm{C}$. Samples were collected from every treatment group every 3 months, and qualities related to processing (bean paste yield and cooked bean hardness), seed moisture content, one-hundred seed weight, seed density, and sample physical properties (electrical conductivity and soluble solid content) were evaluated. Frozen soil storage resulted in the least deterioration of important qualities in adzuki and red kidney beans. We also showed that the electrical conductivity value and soluble solid content of the liquid used for soaking beans can be used to evaluate the processing suitability of cooked beans.
\end{abstract}

Keywords: bean, electrical conductivity, adzuki, natural cold energy, storage

\section{Introduction}

Adzuki beans (Vigna angularis (Willd.) Ohwi et H. Ohashi) are widely grown in East Asian countries such as China and Japan, as well as in the Korean Peninsula (Watanabe, 2000). These beans have high nutritional value. Their protein content is approximately $20 \%$; anthocyanin is abundant in the seed coat, and minerals such as zinc also are abundant (Kagawa, 2010). These beans have traditionally been widely used in Japanese sweets, such as dorayaki and amanatto (a boiled bean paste of adzuki and red kidney beans), and Chinese food items, including jelly and steamed bread (Hatai, 1994).

Red kidney beans (Phaseolus vulgaris L.) were introduced from Latin America to Japan and are grown worldwide. These beans also have high nutritional value and are used in amanatto (Kokubun, 2010).

Hokkaido, in the northernmost region of Japan, produces $86 \%$ of adzuki beans and $94 \%$ of kidney beans in this region (Ministry of Agriculture, Forestry and Fisheries, 2010). Fluctuations in planted acreage and weather conditions affect production. Therefore, it is important to develop storage methods that can ensure a stable supply of high-quality beans (Kato, 2002). Long-term storage of red kidney beans under poor conditions leads to the hard-to-cook (HTC) phenomenon, in which the suitability of the beans for processing declines (Moscoso et al., 1984). Storage at high temperatures $\left(30^{\circ} \mathrm{C}\right)$ also reduced the processing suitability of adzuki beans (Yousif et al., 2002).

In recent years, storage systems that use natural cold energy, rather than freezers that require fuel to operate and cause greenhouse gases to be emitted, have been implemented as a measure to avoid global warming. About 140 such facilities were created in Hokkaido in June 2010 (Planning and Follow-up Group, Development Research Division, Development Administration, 2010). Tsuchiya et al. (1994) reported that storage systems that used natural cold energy are cost-effective, emit less carbon dioxide than freezers, and can reduce the global burden on the environment. In particular, frozen soil storage that does not use power has been used to store vegetables such as potatoes. Storage under these conditions positively affected quality and led to saccharification and sprout inhibition. According to one study, the sugar content of potato stored in frozen soil storage for 4 months 
increased (Tsuchiya \& Ryokai, 1990). However, no studies have reported the effects of frozen soil storage on adzuki and red kidney beans.

In this study, adzuki and red kidney beans were stored under frozen soil storage by using a heat-pipe system; stored in a freezer at $-20^{\circ} \mathrm{C}$, which is known to be an effective method of maintaining food quality during long storage; and maintained at a constant temperature of $25{ }^{\circ} \mathrm{C}$, the usual temperature used for storing beans. Processing suitability values of the beans after storage were compared across the three conditions, in order to reveal the method that most efficiently and conveniently maintained processing suitability.

\section{Materials and Methods}

\subsection{Plant Materials}

Adzuki beans and red kidney beans were harvested in October 2009 at Otofuke, Tokachi, Hokkaido. The beans were maintained at $5{ }^{\circ} \mathrm{C}$ in the dark until the assays were performed in March 2010.

\subsection{Storage Conditions}

The beans were stored under frozen soil storage by using a heat-pipe system (NCE; temp.: $7.1{ }^{\circ} \mathrm{C} \pm 3.4$; relative humidity (RH): $84.8 \% \pm 4.4$, Obihiro City, Hokkaido, Japan); in a freezer at $-20^{\circ} \mathrm{C}$ (FS; RH: $35.5 \% \pm 17.1$ ); and at a constant temperature of $25^{\circ} \mathrm{C}(\mathrm{CS}$; $\mathrm{RH}: 41.3 \% \pm 5.9)$. There was no difference in the temperature or humidity of NCE storage, as measured in a study that spanned 1988 to 1993 (Tsuchiya \& Ryokai, 1996). Temperature and humidity for each storage condition and the outside environment in Hokkaido from March 2010 to February 2011 are shown in Figure 1 (Feng, 2015; Japan Meteorological Agency, 2015). The average temperature from March 2010 to February 2011 in Obihiro City, Hokkaido, Japan, was $7.9{ }^{\circ} \mathrm{C} \pm 10.4$, and relative humidity was $72.6 \% \pm 6.2$. There was no significant difference between the average temperature of NCE storage and the external environment in Obihiro City $(p<0.01)$.

A 1.4-kg portion of the bean sample was placed in polyethylene zipper bags (sealed bags, SB) having dimensions of $270 \mathrm{~mm} \times 280 \mathrm{~mm} \times 0.04 \mathrm{~mm}$ (Daiwa Bussan Co., Ltd.). The average humidity in the SB remained stable during the storage period (RH: adzuki beans, $54.8 \% \pm 7.1$; red kidney beans, $55.0 \% \pm 4.2$ ). The remaining samples were stored in paper bags (PB; tertrapack; tare weight, 280 g; Japan Toyo Paper Co., Ltd.) having dimensions of $830 \mathrm{~mm} \times 417 \mathrm{~mm} \times 0.4 \mathrm{~mm}$.
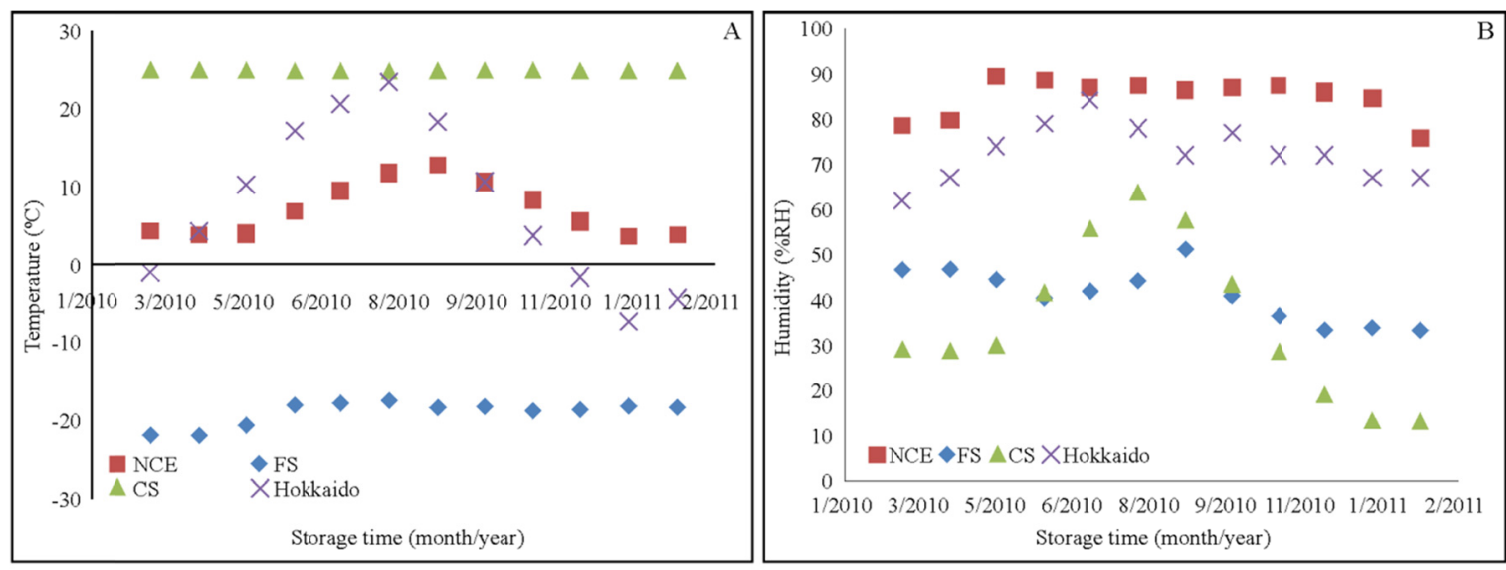

Figure 1. Changes in the temperature and humidity of each storage condition and Hokkaido, Japan from 3/2010 to $2 / 2011$. NCE, natural cold energy (storage in frozen soil with a heat pipe type system ); FS, storage in a freezer at $20^{\circ} \mathrm{C}$; CS, storage at a constant temperature of $25^{\circ} \mathrm{C}$ (Feng, 2015; Japan Meteorological Agency, 2015)

\subsection{Sample Collection}

From March 2010 to May 2011, a sample from each of the three storage conditions was collected every three months $(1.4 \mathrm{~kg} /$ time $)$.

\subsection{Seed Moisture Content}

The seed moisture content (SMC) was measured using the AOAC method (Cunniff, 1995). The decrease in moisture content was measured after the seeds were placed in an oven at $105 \pm 5{ }^{\circ} \mathrm{C}$ for $24 \mathrm{~h}$. 


\subsection{Seed Traits}

Hundred-seed weight (100 SW) and seed density were measured, separately determining the weight of 100 randomly selected seeds of each type of stored beans. In addition, $50 \mathrm{~g}$ beans and $250 \mathrm{~mL}$ deionized water were placed in a $500-\mathrm{mL}$ measuring cylinder, the cylinder was sealed to prevent the entry of air, and the volume was recorded. The weight $(\mathrm{g})$ and volume $(\mathrm{mL})$ values were used to calculate the seed density $(\mathrm{g} / \mathrm{mL})($ Giami \& Okwechime, 1993).

\subsection{Physical Properties and Processing Suitability}

In order to measure the processing suitability of beans, $250 \mathrm{~mL}$ deionized water was added to $50 \mathrm{~g}$ bean seeds. The beans were soaked for $18 \mathrm{~h}$ in an incubator at $25^{\circ} \mathrm{C}$, removed from the soaking liquid, and weighed. The hydration coefficient was calculated (El-Refai et al., 1988). The liquid in which the beans were soaked was collected and filtered, and its electrical conductivity and soluble solid content were measured using a digital electric conductivity meter (DEC-1; Atago Co., Ltd.) and digital pocket refractometer (PAL-Patissier; Atago Co., Ltd.), respectively. Subsequently, the soaked beans were placed in a beaker, $250 \mathrm{~mL}$ deionized water was added, and the beans were autoclaved for $20 \mathrm{~min}$ at $95{ }^{\circ} \mathrm{C}$. They were then cooled at room temperature for $15 \mathrm{~min}$, and the liquid and beans were separated using a bamboo basket. The hardness of the cooked beans was measured using a texture-analyser with a flat 2-mm diameter steel punch that was applied with a crosshead speed of $30 \mathrm{~cm}$ $\min ^{-1}$ (Stable Micro System Co., Ltd.), according to the method described by Reyes-Moreno et al. (1994). A total of $3^{*} 10$ beans were punched individually for each treatment and the mean peak force was calculated in Newtons per seed $\left(\mathrm{N} \mathrm{seed}^{-1}\right)$. Finally, the seed coat of the cooked beans was separated by passing the beans through a $0.5-\mathrm{mm}$ sieve, washing them three times with $1 \mathrm{~L}$ deionized water, and squeezed using a bleached cotton cloth bag to prepare a raw bean paste. The yield of the paste was calculated as follows: bean paste yield $=$ raw bean paste dry weight/bean dry weight $\times 100$.

\subsection{Statistical Analysis}

All the data are expressed as means \pm standard deviation (SD). Analysis of variance (ANOVA) with Tukey's studentized range (HSD) test was conducted using SAS 9.3, and differences were considered significant at $p<$ 0.01 .

\section{Results}

The changes in SMC, seed traits, and processing suitability of adzuki and red kidney beans stored under the three different conditions are shown in Tables 1-4.

\subsection{Seed Moisture Content}

The SMC of red kidney beans was higher than that of adzuki beans stored in PB (Tables 1 and 2). There was almost no difference in the SMC of the two types of beans stored in the SB under the three storage conditions for 15 months. The SMC of both types of beans was greatest when they were stored in PB at NCE for 15 months, and that of beans stored in PB at CS was the smallest. Thus, packaging in SB might suppress the variation in moisture content of both types of beans. 
Table 1. Changes in the seed moisture content, hundred seed weight, seed density, and hydration coefficient of adzuki beans

\begin{tabular}{llll}
\hline $\begin{array}{l}\text { SB } \\
\text { Storage time } \\
\text { (months) }\end{array}$ & \multicolumn{3}{l}{ Seed moisture content $(\%)$} \\
\cline { 2 - 4 } & NCE & FS & CS \\
\hline 0 & $12.3 \pm 1.0 \mathrm{a}$ & $12.3 \pm 1.0 \mathrm{a}$ & $12.3 \pm 1.0 \mathrm{a}$ \\
3 & $11.8 \pm 0.1 \mathrm{a}$ & $12.3 \pm 0.1 \mathrm{a}$ & $11.4 \pm 0.1 \mathrm{a}$ \\
6 & $11.8 \pm 0.1 \mathrm{a}$ & $11.6 \pm 0.1 \mathrm{a}$ & $11.8 \pm 0.0 \mathrm{a}$ \\
9 & $11.8 \pm 0.0 \mathrm{a}$ & $12.2 \pm 0.3 \mathrm{a}$ & $11.9 \pm 0.0 \mathrm{a}$ \\
12 & $12.3 \pm 0.1 \mathrm{a}$ & $12.2 \pm 0.1 \mathrm{a}$ & $11.9 \pm 0.4 \mathrm{a}$ \\
15 & $12.4 \pm 0.5 \mathrm{a}$ & $12.2 \pm 0.1 \mathrm{a}$ & $12.0 \pm 0.1 \mathrm{a}$
\end{tabular}

Hundred seed weight $(\mathrm{g})$

$\begin{array}{llll} & \text { NCE } & \text { FS } & \text { CS } \\ 0 & 13.2 \pm 0.1 \mathrm{a} & 13.2 \pm 0.1 \mathrm{a} & 13.2 \pm 0.1 \mathrm{a} \\ 3 & 13.0 \pm 0.1 \mathrm{a} & 13.3 \pm 0.2 \mathrm{a} & 13.5 \pm 0.3 \mathrm{a} \\ 6 & 13.1 \pm 0.1 \mathrm{a} & 13.2 \pm 0.2 \mathrm{a} & 13.1 \pm 0.2 \mathrm{a} \\ 9 & 13.0 \pm 0.1 \mathrm{a} & 13.1 \pm 0.1 \mathrm{a} & 13.1 \pm 0.2 \mathrm{a} \\ 12 & 13.1 \pm 0.1 \mathrm{a} & 13.1 \pm 0.1 \mathrm{a} & 12.8 \pm 0.2 \mathrm{a} \\ 15 & 12.9 \pm 0.1 \mathrm{a} & 13.1 \pm 0.1 \mathrm{a} & 13.1 \pm 0.1 \mathrm{a}\end{array}$

Seed density $(\mathrm{g} / \mathrm{mL})$

NCE FS CS

$\begin{array}{llll}0 & 1.32 \pm 0.0 \mathrm{a} & 1.32 \pm 0.0 \mathrm{a} & 1.32 \pm 0.0 \mathrm{a} \\ 3 & 1.32 \pm 0.0 \mathrm{a} & 1.29 \pm 0.0 \mathrm{a} & 1.33 \pm 0.0 \mathrm{a} \\ 6 & 1.32 \pm 0.0 \mathrm{a} & 1.31 \pm 0.0 \mathrm{a} & 1.33 \pm 0.0 \mathrm{a} \\ 9 & 1.32 \pm 0.0 \mathrm{a} & 1.32 \pm 0.0 \mathrm{a} & 1.33 \pm 0.0 \mathrm{a} \\ 12 & 1.32 \pm 0.0 \mathrm{a} & 1.32 \pm 0.0 \mathrm{a} & 1.32 \pm 0.0 \mathrm{a} \\ 15 & 1.32 \pm 0.0 \mathrm{a} & 1.32 \pm 0.0 \mathrm{a} & 1.32 \pm 0.0 \mathrm{a}\end{array}$

Hydration coefficient

$\begin{array}{llll} & \text { NCE } & \text { FS } & \text { CS } \\ 0 & 235 \pm 4 a & 235 \pm 4 a & 235 \pm 4 a \\ 3 & 239 \pm 9 a & 246 \pm 5 a & 243 \pm 5 a \\ 6 & 236 \pm 5 a & 235 \pm 4 a & 237 \pm 6 a \\ 9 & 238 \pm 0 a & 236 \pm 0 a & 237 \pm 1 \mathrm{a} \\ 12 & 237 \pm 0 a & 236 \pm 0 a & 237 \pm 2 a \\ 15 & 237 \pm 0 a & 236 \pm 0 a & 236 \pm 0 a\end{array}$

PB

Storage time Seed moisture content (\%)

$\begin{array}{llll}\text { (months) } & \text { NCE } & \text { FS } & \text { CS } \\ 0 & 12.3 \pm 1.0 \mathrm{a} & 12.3 \pm 1.0 \mathrm{a} & 12.3 \pm 1.0 \mathrm{a} \\ 3 & 14.1 \pm 0.2 \mathrm{a}^{*} & 12.4 \pm 0.0 \mathrm{ab} & 8.6 \pm 0.2 \mathrm{c}^{*} \\ 6 & 15.3 \pm 0.3 \mathrm{a}^{*} & 12.4 \pm 0.1 \mathrm{~b} & 11.4 \pm 0.1 \mathrm{c}^{*} \\ 9 & 15.9 \pm 0.1 \mathrm{a}^{*} & 12.9 \pm 0.1 \mathrm{~b} & 9.1 \pm 0.1 \mathrm{c}^{*}\end{array}$




\begin{tabular}{llll}
12 & $16.5 \pm 0.1 \mathrm{a}^{*}$ & $13.3 \pm 0.2 \mathrm{~b}$ & $7.4 \pm 0.1 \mathrm{c}^{*}$ \\
15 & $15.6 \pm 0.2 \mathrm{a}^{*}$ & $12.69 \pm 0.1 \mathrm{~b}^{*}$ & $5.7 \pm 0.1 \mathrm{c}^{*}$ \\
& \multicolumn{1}{l}{ Hundred seed weight $(\mathrm{g})$} & \\
& NCE & FS & CS \\
0 & $13.2 \pm 0.1 \mathrm{a}$ & $13.2 \pm 0.1 \mathrm{a}$ & $13.2 \pm 0.1 \mathrm{a}$ \\
3 & $13.9 \pm 0.2 \mathrm{a}$ & $13.4 \pm 0.2 \mathrm{ab}$ & $12.6 \pm 0.2 \mathrm{~b}$ \\
6 & $13.8 \pm 0.2 \mathrm{a}$ & $13.2 \pm 0.1 \mathrm{ab}$ & $13.1 \pm 0.1 \mathrm{~b}$ \\
9 & $13.8 \pm 0.3 \mathrm{a}$ & $13.2 \pm 0.2 \mathrm{a}$ & $12.4 \pm 0.1 \mathrm{~b}$ \\
12 & $13.8 \pm 0.2 \mathrm{a}$ & $13.2 \pm 0.1 \mathrm{~b}$ & $12.1 \pm 0.0 \mathrm{c}$ \\
15 & $14.0 \pm 0.1 \mathrm{a}$ & $13.1 \pm 0.1 \mathrm{~b}$ & $12.3 \pm 0.1 \mathrm{c}$
\end{tabular}

Seed density $(\mathrm{g} / \mathrm{mL})$

$\begin{array}{llll} & \text { NCE } & \text { FS } & \text { CS } \\ 0 & 1.32 \pm 0.0 \mathrm{a} & 1.32 \pm 0.0 \mathrm{a} & 1.32 \pm 0.0 \mathrm{a} \\ 3 & 1.33 \pm 0.0 \mathrm{a} & 1.33 \pm 0.0 \mathrm{a} & 1.35 \pm 0.0 \mathrm{a} \\ 6 & 1.32 \pm 0.0 \mathrm{a} & 1.28 \pm 0.0 \mathrm{a} & 1.31 \pm 0.0 \mathrm{a} \\ 9 & 1.32 \pm 0.0 \mathrm{a} & 1.32 \pm 0.0 \mathrm{a} & 1.32 \pm 0.0 \mathrm{a} \\ 12 & 1.32 \pm 0.0 \mathrm{a} & 1.32 \pm 0.0 \mathrm{a} & 1.32 \pm 0.0 \mathrm{a} \\ 15 & 1.32 \pm 0.0 \mathrm{a} & 1.32 \pm 0.0 \mathrm{a} & 1.32 \pm 0.0 \mathrm{a}\end{array}$

Hydration coefficient

NCE $\quad$ FS CS

$\begin{array}{llll}0 & 235 \pm 4 \mathrm{a} & 235 \pm 4 \mathrm{a} & 235 \pm 4 \mathrm{a} \\ 3 & 240 \pm 9 \mathrm{a} & 241 \pm 10 \mathrm{a} & 243 \pm 7 \mathrm{a} \\ 6 & 239 \pm 7 \mathrm{a} & 236 \pm 5 \mathrm{a} & 236 \pm 5 \mathrm{a} \\ 9 & 239 \pm 1 \mathrm{a} & 239 \pm 3 \mathrm{a} & 236 \pm 7 \mathrm{a} \\ 12 & 240 \pm 2 \mathrm{a} & 238 \pm 0 \mathrm{a} & 236 \pm 5 \mathrm{a} \\ 15 & 240 \pm 2 \mathrm{a} & 238 \pm 0 \mathrm{a} & 234 \pm 5 \mathrm{a}\end{array}$

NCE, natural cold energy (storage in frozen soil with a heat pipe type system ); FS, storage in a freezer at $20{ }^{\circ} \mathrm{C}$; $\mathrm{CS}$, storage at a constant temperature of $25^{\circ} \mathrm{C}$; SB, sealed bags; PB, paper bags. Data are expressed as means \pm $\mathrm{SD}(\mathrm{n}=3)$. The values with different superscript letters in the same row are significantly different among the storage conditions $(\mathrm{p}<0.01)$. The superscript $*$ indicates significant difference in the data presented in the columns compared with the values for 0 month $(\mathrm{p}<0.01)$.

\subsection{Seed Traits}

The $100 \mathrm{SW}$ of adzuki beans placed in SB was not significantly different across the three storage conditions (12.1-14.0 g), whereas that of adzuki beans stored at NCE in PB was the greatest, indicating the highest retention of moisture content (Table 1). On the other hand, there was no significant difference in the $100 \mathrm{SW}$ of red kidney beans stored under the three storage conditions in SB and PB (67.9-72.5 g; Table 2).

There were no changes in seed density of adzuki and red kidney beans, irrespective of the storage conditions or packaging methods used (Tables 1 and 2). 
Table 2. Changes in the seed moisture content, hundred seed weight, seed density, and hydration coefficient of red kidney

\begin{tabular}{|c|c|c|c|}
\hline \multicolumn{4}{|l|}{ SB } \\
\hline \multirow{2}{*}{$\begin{array}{l}\text { Storage time } \\
\text { (months) }\end{array}$} & \multicolumn{3}{|c|}{ Seed moisture content $(\%)$} \\
\hline & NCE & FS & $\mathrm{CS}$ \\
\hline 0 & $13.2 \pm 0.2 \mathrm{a}$ & $13.2 \pm 0.2 \mathrm{a}$ & $13.2 \pm 0.2 \mathrm{a}$ \\
\hline 3 & $11.3 \pm 0.2 \mathrm{a}^{*}$ & $11.2 \pm 0.5 \mathrm{a}$ & $11.5 \pm 0.3 \mathrm{a}^{*}$ \\
\hline 6 & $12.0 \pm 0.2 \mathrm{a}^{*}$ & $12.5 \pm 0.2 \mathrm{a}$ & $12.2 \pm 0.2 \mathrm{a}^{*}$ \\
\hline 9 & $12.2 \pm 0.1 \mathrm{a}^{*}$ & $12.8 \pm 0.1 \mathrm{a}$ & $13.3 \pm 0.2 \mathrm{a}$ \\
\hline 12 & $13.6 \pm 0.7 \mathrm{a}$ & $13.3 \pm 0.8 \mathrm{a}$ & $13.1 \pm 0.1 \mathrm{a}$ \\
\hline \multirow[t]{3}{*}{15} & $11.8 \pm 0.2 \mathrm{a}^{*}$ & $11.8 \pm 0.5 \mathrm{a}$ & $12.0 \pm 0.1 \mathrm{a}^{*}$ \\
\hline & \multicolumn{3}{|c|}{ Hundred seed weight (g) } \\
\hline & $\mathrm{NCE}$ & FS & CS \\
\hline 0 & $67.9 \pm 3.3 \mathrm{a}$ & $67.9 \pm 3.3 \mathrm{a}$ & $67.9 \pm 3.3 \mathrm{a}$ \\
\hline 3 & $70.9 \pm 0.7 \mathrm{a}$ & $68.6 \pm 2.0 \mathrm{a}$ & $70.4 \pm 1.2 \mathrm{a}$ \\
\hline 6 & $70.5 \pm 0.8 \mathrm{a}$ & $69.2 \pm 0.8 \mathrm{a}$ & $69.3 \pm 2.0 \mathrm{a}$ \\
\hline 9 & $70.6 \pm 0.8 \mathrm{a}$ & $67.9 \pm 2.0 \mathrm{a}$ & $69.0 \pm 1.9 \mathrm{a}$ \\
\hline 12 & $69.9 \pm 1.1 \mathrm{a}$ & $69.5 \pm 1.4 \mathrm{a}$ & $67.8 \pm 0.6 \mathrm{a}$ \\
\hline \multirow[t]{3}{*}{15} & $68.7 \pm 0.8 \mathrm{a}$ & $67.8 \pm 1.4 \mathrm{a}$ & $70.4 \pm 1.2 \mathrm{a}$ \\
\hline & \multicolumn{3}{|c|}{ Seed density $(\mathrm{g} / \mathrm{mL})$} \\
\hline & NCE & FS & CS \\
\hline 0 & $1.27 \pm 0.0 \mathrm{a}$ & $1.27 \pm 0.0 \mathrm{a}$ & $1.27 \pm 0.0 \mathrm{a}$ \\
\hline 3 & $1.25 \pm 0.0 \mathrm{a}$ & $1.25 \pm 0.0 \mathrm{a}$ & $1.26 \pm 0.0 \mathrm{a}$ \\
\hline 6 & $1.25 \pm 0.0 \mathrm{a}$ & $1.26 \pm 0.0 \mathrm{a}$ & $1.28 \pm 0.0 \mathrm{a}$ \\
\hline 9 & $1.25 \pm 0.0 \mathrm{a}$ & $1.25 \pm 0.0 \mathrm{a}$ & $1.25 \pm 0.0 \mathrm{a}$ \\
\hline 12 & $1.25 \pm 0.0 \mathrm{a}$ & $1.25 \pm 0.0 \mathrm{a}$ & $1.25 \pm 0.0 \mathrm{a}$ \\
\hline \multirow[t]{3}{*}{15} & $1.25 \pm 0.0 \mathrm{a}$ & $1.25 \pm 0.0 \mathrm{a}$ & $1.25 \pm 0.0 \mathrm{a}$ \\
\hline & \multicolumn{3}{|c|}{ Hydration coefficient } \\
\hline & NCE & FS & $\mathrm{CS}$ \\
\hline 0 & $225 \pm 1 \mathrm{a}$ & $225 \pm 1 \mathrm{a}$ & $225 \pm 1 \mathrm{a}$ \\
\hline 3 & $226 \pm 1 \mathrm{a}$ & $227 \pm 1 \mathrm{a}$ & $224 \pm 2 \mathrm{a}$ \\
\hline 6 & $227 \pm 1 \mathrm{a}$ & $227 \pm 1 \mathrm{a}$ & $225 \pm 1 \mathrm{a}$ \\
\hline 9 & $228 \pm 0 \mathrm{a}$ & $224 \pm 1 \mathrm{a}$ & $228 \pm 4 a$ \\
\hline 12 & $229 \pm 5 \mathrm{a}$ & $229 \pm 3 \mathrm{a}$ & $229 \pm 1 \mathrm{a}$ \\
\hline 15 & $228 \pm 3 a$ & $228 \pm 3 a$ & $228 \pm 0 \mathrm{a}$ \\
\hline \multicolumn{4}{|l|}{ PB } \\
\hline Storage time & \multicolumn{3}{|c|}{ Seed moisture content (\%) } \\
\hline (months) & NCE & FS & $\mathrm{CS}$ \\
\hline 0 & $13.2 \pm 0.2 \mathrm{a}$ & $13.2 \pm 0.2 \mathrm{a}$ & $13.2 \pm 0.2 \mathrm{a}$ \\
\hline 3 & $14.6 \pm 0.3 \mathrm{a}$ & $12.5 \pm 0.3 b$ & $7.2 \pm 0.5 \mathrm{c}$ \\
\hline 6 & $17.1 \pm 0.7 \mathrm{a}^{*}$ & $13.2 \pm 0.1 \mathrm{~b}$ & $11.5 \pm 0.1 \mathrm{~b}$ \\
\hline 9 & $20.2 \pm 0.8 \mathrm{a}^{*}$ & $12.9 \pm 1.4 \mathrm{~b}$ & $9.3 \pm 3.5 b$ \\
\hline
\end{tabular}




\begin{tabular}{llll}
12 & $20.3 \pm 0.3 \mathrm{a}^{*}$ & $13.7 \pm 1.6 \mathrm{~b}$ & $10.2 \pm 0.2 \mathrm{~b}$ \\
15 & $19.4 \pm 0.6 \mathrm{a} *$ & $11.2 \pm 0.5 \mathrm{~b}$ & $9.8 \pm 0.9 \mathrm{~b}$ \\
& \multicolumn{1}{l}{ Hundred seed weight $(\mathrm{g})$} & \\
& NCE & FS & CS \\
0 & $67.9 \pm 3.3 \mathrm{a}$ & $67.9 \pm 3.3 \mathrm{a}$ & $67.9 \pm 3.3 \mathrm{a}$ \\
3 & $72.5 \pm 1.9 \mathrm{a}$ & $70.8 \pm 2.0 \mathrm{a}$ & $70.9 \pm 1.1 \mathrm{a}$ \\
6 & $70.1 \pm 1.0 \mathrm{a}$ & $69.4 \pm 1.1 \mathrm{a}$ & $71.5 \pm 0.6 \mathrm{a}$ \\
9 & $69.9 \pm 0.9 \mathrm{a}$ & $66.2 \pm 1.7 \mathrm{a}$ & $67.9 \pm 1.6 \mathrm{a}$ \\
12 & $70.5 \pm 1.3 \mathrm{a}$ & $68.5 \pm 0.8 \mathrm{a}$ & $68.8 \pm 0.7 \mathrm{a}$ \\
15 & $68.7 \pm 0.8 \mathrm{a}$ & $69.7 \pm 0.8 \mathrm{a}$ & $70.7 \pm 1.7 \mathrm{a}$
\end{tabular}

Seed density $(\mathrm{g} / \mathrm{mL})$

NCE

$1.27 \pm 0.0 \mathrm{a}$

$1.25 \pm 0.0 \mathrm{a}$

$1.25 \pm 0.0 \mathrm{a}$

$1.25 \pm 0.0 \mathrm{a}$

$1.25 \pm 0.0 \mathrm{a}$

$1.25 \pm 0.0 \mathrm{a}$

Hydration coefficien

NCE

0

3

6

9

12

15
FS

$1.27 \pm 0.0 \mathrm{a}$

$1.25 \pm 0.0 \mathrm{a}$

$1.26 \pm 0.0 \mathrm{a}$

$1.25 \pm 0.0 \mathrm{a}$

$1.25 \pm 0.0 \mathrm{a}$

$1.25 \pm 0.0 \mathrm{a}$

FS

$225 \pm 1 \mathrm{a}$

$227 \pm 2 \mathrm{a}$

$226 \pm 1 \mathrm{a}$

$233 \pm 1 \mathrm{a}$

$234 \pm 3 a$

$226 \pm 2 \mathrm{a}$
CS

$1.27 \pm 0.0 \mathrm{a}$

$1.26 \pm 0.0 \mathrm{a}$

$1.26 \pm 0.0 \mathrm{a}$

$1.25 \pm 0.0 \mathrm{a}$

$1.25 \pm 0.0 \mathrm{a}$

$1.25 \pm 0.0 \mathrm{a}$

NCE, natural cold energy (storage in frozen soil with a heat pipe type system ); FS, storage in a freezer at $20{ }^{\circ} \mathrm{C}$; $\mathrm{CS}$, storage at a constant temperature of $25^{\circ} \mathrm{C}$; SB, sealed bags; $\mathrm{PB}$, paper bags. Data are expressed as means \pm $\mathrm{SD}(\mathrm{n}=3)$. The values with different superscript letters in the same row are significantly different among the storage conditions $(\mathrm{p}<0.01)$. The superscript $*$ indicates significant difference in the data presented in the columns compared with the values for 0 month $(\mathrm{p}<0.01)$.

\subsection{Physical Properties and Processing Suitability}

The hydration coefficient, electrical conductivity, soluble solid content, and hardness of beans stored under NCE and FS conditions were not significantly different between the two types of beans (Tables 3 and 4). The values of all the physical properties, except the hydration coefficient of adzuki and red kidney beans stored at CS, significantly increased at 12 and 9 months $(p<0.01)$, respectively.

There was no significant difference in the paste yield of both the beans stored under NCE and FS conditions for 15 months (56.81-60.97\% and 61.71-63.53\%, respectively), whereas that of both the types of beans stored under CS conditions decreased significantly ( $p<0.01$; Tables 3 and 4).

\subsection{Correlation Between Physical Properties and Processing Suitability}

The SAS software package was used to calculate the correlation coefficients between physical properties and processing suitability. The hardness of adzuki beans was strongly positively correlated with both electrical conductivity and the soluble solid content of the soaking liquid $\left(\mathrm{r}_{\mathrm{SB}}=0.983, \mathrm{r}_{\mathrm{PB}}=0.935, \mathrm{r}_{\mathrm{SB}}=0.943, \mathrm{r}_{\mathrm{PB}}=0.944\right.$; Figure 2). The correlation coefficients between physical properties and processing suitability of red kidney beans 
were similar to those of adzuki beans, as shown in Figure 3.

Table 3. Changes in physical properties and processing suitability of adzuki beans stored at different storage conditions

\begin{tabular}{|c|c|c|c|}
\hline \multicolumn{4}{|l|}{ SB } \\
\hline \multirow{2}{*}{$\begin{array}{l}\text { Storage time } \\
\text { (months) }\end{array}$} & \multicolumn{3}{|c|}{ Electrical conductivity $(\mu \mathrm{S} / \mathrm{cm})$} \\
\hline & NCE & FS & $\mathrm{CS}$ \\
\hline 0 & $900 \pm 0 \mathrm{a}$ & $900 \pm 0 \mathrm{a}$ & $900 \pm 0 \mathrm{a}$ \\
\hline 3 & $900 \pm 0 \mathrm{a}$ & $900 \pm 0 \mathrm{a}$ & $900 \pm 0 \mathrm{a}$ \\
\hline 6 & $900 \pm 0 \mathrm{a}$ & $900 \pm 0 \mathrm{a}$ & $900 \pm 0 \mathrm{a}$ \\
\hline 9 & $900 \pm 0 \mathrm{~b}$ & $900 \pm 0 \mathrm{~b}$ & $1067 \pm 58 \mathrm{a}^{*}$ \\
\hline 12 & $900 \pm 0 \mathrm{~b}$ & $900 \pm 0 \mathrm{~b}$ & $1300 \pm 0 \mathrm{a}^{*}$ \\
\hline \multirow[t]{3}{*}{15} & $900 \pm 0 \mathrm{~b}$ & $900 \pm 0 \mathrm{~b}$ & $1467 \pm 58 \mathrm{a}^{*}$ \\
\hline & \multicolumn{3}{|c|}{ Soluble solid content (Brix\%) } \\
\hline & NCE & FS & CS \\
\hline 0 & $0.4 \pm 0.1 \mathrm{a}$ & $0.4 \pm 0.1 \mathrm{a}$ & $0.4 \pm 0.1 \mathrm{a}$ \\
\hline 3 & $0.4 \pm 0.0 \mathrm{a}$ & $0.4 \pm 0.0 \mathrm{a}$ & $0.4 \pm 0.0 \mathrm{a}$ \\
\hline 6 & $0.4 \pm 0.0 \mathrm{a}$ & $0.4 \pm 0.0 \mathrm{a}$ & $0.4 \pm 0.0 \mathrm{a}$ \\
\hline 9 & $0.4 \pm 0.0 \mathrm{a}$ & $0.4 \pm 0.0 \mathrm{a}$ & $0.4 \pm 0.0 \mathrm{a}$ \\
\hline 12 & $0.4 \pm 0.0 \mathrm{~b}$ & $0.4 \pm 0.0 \mathrm{~b}$ & $0.6 \pm 0.0 \mathrm{a}^{*}$ \\
\hline \multirow[t]{3}{*}{15} & $0.4 \pm 0.0 \mathrm{~b}$ & $0.4 \pm 0.0 \mathrm{~b}$ & $0.6 \pm 0.0 \mathrm{a}^{*}$ \\
\hline & \multicolumn{3}{|c|}{ Hardness of cooked beans (N/seed) } \\
\hline & NCE & FS & $\mathrm{CS}$ \\
\hline 0 & $0.529 \pm 0.0 \mathrm{a}$ & $0.529 \pm 0.0 \mathrm{a}$ & $0.529 \pm 0.0 \mathrm{a}$ \\
\hline 3 & $0.534 \pm 0.0 \mathrm{a}$ & $0.520 \pm 0.0 \mathrm{a}$ & $0.505 \pm 0.0 \mathrm{a}$ \\
\hline 6 & $0.551 \pm 0.1 \mathrm{a}$ & $0.535 \pm 0.1 \mathrm{a}$ & $0.702 \pm 0.1 \mathrm{a}^{*}$ \\
\hline 9 & $0.538 \pm 0.0 \mathrm{~b}$ & $0.537 \pm 0.1 \mathrm{~b}$ & $1.140 \pm 0.2 \mathrm{a}^{*}$ \\
\hline 12 & $0.548 \pm 0.1 \mathrm{~b}$ & $0.505 \pm 0.1 \mathrm{~b}$ & $2.724 \pm 0.1 \mathrm{a}^{*}$ \\
\hline \multirow[t]{3}{*}{15} & $0.540 \pm 0.1 \mathrm{~b}$ & $0.517 \pm 0.0 \mathrm{~b}$ & $3.739 \pm 0.1 \mathrm{a}^{*}$ \\
\hline & \multicolumn{3}{|c|}{ Yield of bean paste $(\%)$} \\
\hline & $\mathrm{NCE}$ & FS & $\mathrm{CS}$ \\
\hline 0 & $59.43 \pm 2.3 \mathrm{a}$ & $59.43 \pm 2.3 \mathrm{a}$ & $59.43 \pm 2.3 \mathrm{a}$ \\
\hline 3 & $59.13 \pm 3.2 \mathrm{a}$ & $59.76 \pm 2.5 \mathrm{a}$ & $58.68 \pm 2.7 \mathrm{a}$ \\
\hline 6 & $59.47 \pm 3.0 \mathrm{a}$ & $59.83 \pm 4.0 \mathrm{a}$ & $58.47 \pm 2.1 \mathrm{a}$ \\
\hline 9 & $60.97 \pm 2.3 \mathrm{a}$ & $60.83 \pm 2.6 \mathrm{a}$ & $52.73 \pm 1.8 \mathrm{a}$ \\
\hline 12 & $57.87 \pm 1.6 \mathrm{a}$ & $60.40 \pm 1.0 \mathrm{a}$ & $38.06 \pm 2.9 b^{*}$ \\
\hline 15 & $58.27 \pm 2.0 \mathrm{a}$ & $58.00 \pm 3.3 \mathrm{a}$ & $19.63 \pm 1.3 \mathrm{c}^{*}$ \\
\hline
\end{tabular}

\section{PB}

Storage time

Electrical conductivity $(\mu \mathrm{S} / \mathrm{cm})$

(months)

NCE

$900 \pm 0 \mathrm{a}$

FS

CS

0

$900 \pm 0 \mathrm{~b}$

$900 \pm 0 \mathrm{a}$

$900 \pm 0 \mathrm{a}$

3

$900 \pm 0 \mathrm{~b}$

$1333 \pm 58 \mathrm{a}$ 


\begin{tabular}{|c|c|c|c|}
\hline 6 & $900 \pm 0 \mathrm{~b}$ & $900 \pm 0 \mathrm{~b}$ & $1400 \pm 100 \mathrm{a}$ \\
\hline 9 & $900 \pm 0 \mathrm{~b}$ & $900 \pm 0 \mathrm{~b}$ & $1933 \pm 58 \mathrm{a}^{*}$ \\
\hline 12 & $900 \pm 0 \mathrm{~b}$ & $900 \pm 0 \mathrm{~b}$ & $2233 \pm 58 \mathrm{a}^{*}$ \\
\hline \multirow[t]{3}{*}{15} & $900 \pm 0 \mathrm{~b}$ & $900 \pm 0 \mathrm{~b}$ & $2600 \pm 100 a^{*}$ \\
\hline & \multicolumn{3}{|c|}{ Soluble solid content (Brix\%) } \\
\hline & NCE & FS & $\mathrm{CS}$ \\
\hline 0 & $0.4 \pm 0.1 \mathrm{a}$ & $0.4 \pm 0.1 \mathrm{a}$ & $0.4 \pm 0.1 \mathrm{a}$ \\
\hline 3 & $0.4 \pm 0.0 \mathrm{a}$ & $0.4 \pm 0.0 \mathrm{a}$ & $0.5 \pm 0.0 \mathrm{a}$ \\
\hline 6 & $0.4 \pm 0.0 \mathrm{a}$ & $0.4 \pm 0.0 \mathrm{a}$ & $0.5 \pm 0.0 \mathrm{a}$ \\
\hline 9 & $0.4 \pm 0.0 \mathrm{~b}$ & $0.4 \pm 0.0 \mathrm{~b}$ & $0.7 \pm 0.0 \mathrm{a}$ \\
\hline 12 & $0.4 \pm 0.0 \mathrm{~b}$ & $0.4 \pm 0.0 \mathrm{~b}$ & $0.8 \pm 0.1 \mathrm{a}^{*}$ \\
\hline \multirow[t]{3}{*}{15} & $0.4 \pm 0.0 \mathrm{~b}$ & $0.4 \pm 0.0 \mathrm{~b}$ & $0.9 \pm 0.0 \mathrm{a}^{*}$ \\
\hline & \multicolumn{3}{|c|}{ Hardness of cooked beans (N/seed) } \\
\hline & NCE & FS & $\mathrm{CS}$ \\
\hline 0 & $0.529 \pm 0.0 \mathrm{a}$ & $0.529 \pm 0.0 \mathrm{a}$ & $0.529 \pm 0.0 \mathrm{a}$ \\
\hline 3 & $0.532 \pm 0.0 \mathrm{a}$ & $0.518 \pm 0.0 \mathrm{a}$ & $0.542 \pm 0.0 \mathrm{a}$ \\
\hline 6 & $0.530 \pm 0.1 \mathrm{a}$ & $0.513 \pm 0.1 \mathrm{a}$ & $0.539 \pm 0.0 \mathrm{a}$ \\
\hline 9 & $0.540 \pm 0.0 \mathrm{~b}$ & $0.512 \pm 0.1 \mathrm{~b}$ & $0.949 \pm 0.1 \mathrm{a}^{*}$ \\
\hline 12 & $0.528 \pm 0.0 \mathrm{~b}$ & $0.496 \pm 0.1 b$ & $1.062 \pm 0.1 \mathrm{a}^{*}$ \\
\hline 15 & $0.526 \pm 0.0 \mathrm{~b}$ & $0.500 \pm 0.0 \mathrm{~b}$ & $1.139 \pm 0.1 \mathrm{a}^{*}$ \\
\hline
\end{tabular}

Yield of bean paste (\%)

NCE $\quad$ FS CS

$\begin{array}{llll}0 & 59.43 \pm 2.3 \mathrm{a} & 59.43 \pm 2.3 \mathrm{a} & 59.43 \pm 2.3 \mathrm{a} \\ 3 & 58.74 \pm 2.9 \mathrm{a} & 58.60 \pm 4.4 \mathrm{a} & 57.63 \pm 2.6 \mathrm{a} \\ 6 & 59.93 \pm 4.1 \mathrm{a} & 59.31 \pm 3.0 \mathrm{a} & 57.96 \pm 2.5 \mathrm{a} \\ 9 & 58.85 \pm 1.8 \mathrm{a} & 59.83 \pm 1.9 \mathrm{a} & 53.17 \pm 3.3 \mathrm{a}^{*} \\ 12 & 57.88 \pm 2.1 \mathrm{a} & 56.81 \pm 1.3 \mathrm{a} & 46.18 \pm 3.2 \mathrm{~b}^{*} \\ 15 & 58.55 \pm 1.1 \mathrm{a} & 57.53 \pm 2.6 \mathrm{a} & 44.10 \pm 0.8 \mathrm{~b}^{*}\end{array}$

$\mathrm{NCE}$, natural cold energy (storage in frozen soil with a heat pipe type system ); FS, storage in a freezer at $20{ }^{\circ} \mathrm{C}$; $\mathrm{CS}$, storage at a constant temperature of $25^{\circ} \mathrm{C} ; \mathrm{SB}$, sealed bags; $\mathrm{PB}$, paper bags. Data are expressed as means \pm $\mathrm{SD}(\mathrm{n}=3)$. The values with different superscript letters in the same row are significantly different among the storage conditions $(\mathrm{p}<0.01)$. The superscript $*$ indicates significant difference in the data presented in the columns compared with the values for 0 month $(\mathrm{p}<0.01)$. 
Table 4. Changes in the physical properties and processing suitability of red kidney beans stored at different storage conditions

\begin{tabular}{|c|c|c|c|}
\hline \multicolumn{4}{|l|}{ SB } \\
\hline \multirow{2}{*}{$\begin{array}{l}\text { Storage time } \\
\text { (months) }\end{array}$} & \multicolumn{3}{|c|}{ Electrical conductivity $(\mu \mathrm{S} / \mathrm{cm})$} \\
\hline & NCE & FS & $\mathrm{CS}$ \\
\hline 0 & $800 \pm 0 \mathrm{a}$ & $800 \pm 0 \mathrm{a}$ & $800 \pm 0 \mathrm{a}$ \\
\hline 3 & $800 \pm 0 \mathrm{a}$ & $833 \pm 58 \mathrm{a}$ & $800 \pm 0 \mathrm{a}$ \\
\hline 6 & $800 \pm 0 \mathrm{a}$ & $800 \pm 0 \mathrm{a}$ & $800 \pm 0 \mathrm{a}$ \\
\hline 9 & $800 \pm 0 \mathrm{~b}$ & $800 \pm 0 \mathrm{~b}$ & $900 \pm 0 a^{*}$ \\
\hline 12 & $800 \pm 0 \mathrm{~b}$ & $800 \pm 0 \mathrm{~b}$ & $900 \pm 0 \mathrm{a}^{*}$ \\
\hline \multirow[t]{3}{*}{15} & $800 \pm 0 \mathrm{~b}$ & $800 \pm 0 \mathrm{~b}$ & $1100 \pm 0 a^{*}$ \\
\hline & \multicolumn{3}{|c|}{ Soluble solid content (Brix\%) } \\
\hline & $\mathrm{NCE}$ & FS & $\mathrm{CS}$ \\
\hline 0 & $0.4 \pm 0.1 \mathrm{a}$ & $0.4 \pm 0.1 \mathrm{a}$ & $0.4 \pm 0.1 \mathrm{a}$ \\
\hline 3 & $0.4 \pm 0.0 \mathrm{~b}$ & $0.4 \pm 0.0 \mathrm{~b}$ & $0.5 \pm 0.0 \mathrm{a}^{*}$ \\
\hline 6 & $0.4 \pm 0.0 \mathrm{a}$ & $0.4 \pm 0.0 \mathrm{a}$ & $0.4 \pm 0.0 \mathrm{a}$ \\
\hline 9 & $0.4 \pm 0.0 \mathrm{~b}$ & $0.4 \pm 0.0 \mathrm{~b}$ & $0.5 \pm 0.0 \mathrm{a}^{*}$ \\
\hline 12 & $0.4 \pm 0.0 \mathrm{~b}$ & $0.4 \pm 0.0 \mathrm{~b}$ & $0.5 \pm 0.0 \mathrm{a}^{*}$ \\
\hline \multirow[t]{3}{*}{15} & $0.4 \pm 0.0 \mathrm{~b}$ & $0.4 \pm 0.0 \mathrm{~b}$ & $0.5 \pm 0.0 \mathrm{a}^{*}$ \\
\hline & \multicolumn{3}{|c|}{ Hardness of cooked beans (N/seed) } \\
\hline & NCE & FS & $\mathrm{CS}$ \\
\hline 0 & $1.127 \pm 0.0 \mathrm{a}$ & $1.127 \pm 0.0 \mathrm{a}$ & $1.127 \pm 0.0 \mathrm{a}$ \\
\hline 3 & $1.151 \pm 0.1 \mathrm{a}$ & $1.119 \pm 0.1 \mathrm{a}$ & $1.175 \pm 0.3 \mathrm{a}$ \\
\hline 6 & $1.117 \pm 0.1 \mathrm{a}$ & $1.107 \pm 0.1 \mathrm{a}$ & $1.184 \pm 0.2 \mathrm{a}$ \\
\hline 9 & $1.113 \pm 0.1 b$ & $1.064 \pm 0.1 \mathrm{~b}$ & $3.826 \pm 0.2 \mathrm{a}^{*}$ \\
\hline 12 & $1.131 \pm 0.1 b$ & $1.085 \pm 0.0 \mathrm{~b}$ & $3.939 \pm 0.2 \mathrm{a}^{*}$ \\
\hline \multirow[t]{3}{*}{15} & $1.109 \pm 0.2 b$ & $1.055 \pm 0.2 \mathrm{~b}$ & $5.968 \pm 0.5 \mathrm{a}^{*}$ \\
\hline & \multicolumn{3}{|c|}{ Yield of bean paste $(\%)$} \\
\hline & $\mathrm{NCE}$ & FS & $\mathrm{CS}$ \\
\hline 0 & $63.53 \pm 2.8 \mathrm{a}$ & $63.53 \pm 2.8 \mathrm{a}$ & $63.53 \pm 2.8 \mathrm{a}$ \\
\hline 3 & $62.70 \pm 4.1 \mathrm{a}$ & $62.51 \pm 4.4 \mathrm{a}$ & $61.06 \pm 4.7 \mathrm{a}$ \\
\hline 6 & $62.56 \pm 2.3 \mathrm{a}$ & $62.02 \pm 2.7 \mathrm{a}$ & $61.04 \pm 3.0 \mathrm{a}$ \\
\hline 9 & $61.92 \pm 2.6 \mathrm{a}$ & $62.04 \pm 3.3 \mathrm{a}$ & $43.03 \pm 3.6 b^{*}$ \\
\hline 12 & $61.90 \pm 2.9 \mathrm{a}$ & $62.84 \pm 3.0 \mathrm{a}$ & $41.30 \pm 1.3 b^{*}$ \\
\hline 15 & $62.09 \pm 1.3 \mathrm{a}$ & $62.84 \pm 3.0 \mathrm{a}$ & $26.13 \pm 0.8 b^{*}$ \\
\hline \multicolumn{4}{|l|}{ PB } \\
\hline Storage time & \multicolumn{3}{|c|}{ Electrical conductivity $(\mu \mathrm{S} / \mathrm{cm})$} \\
\hline (months) & NCE & FS & $\mathrm{CS}$ \\
\hline 0 & $800 \pm 0 \mathrm{a}$ & $800 \pm 0 \mathrm{a}$ & $800 \pm 0 \mathrm{a}$ \\
\hline 3 & $800 \pm 0 \mathrm{a}$ & $800 \pm 0 \mathrm{a}$ & $933 \pm 58 \mathrm{a}$ \\
\hline 6 & $800 \pm 0 \mathrm{a}$ & $800 \pm 0 \mathrm{a}$ & $800 \pm 0 \mathrm{a}$ \\
\hline 9 & $800 \pm 0 \mathrm{~b}$ & $800 \pm 0 \mathrm{~b}$ & $1100 \pm 0 a^{*}$ \\
\hline
\end{tabular}




\begin{tabular}{|c|c|c|c|}
\hline 12 & $800 \pm 0 \mathrm{~b}$ & $800 \pm 0 \mathrm{~b}$ & $1100 \pm 0 \mathrm{a}^{*}$ \\
\hline \multirow[t]{3}{*}{15} & $800 \pm 0 \mathrm{~b}$ & $800 \pm 0 \mathrm{~b}$ & $1000 \pm 0 a^{*}$ \\
\hline & \multicolumn{3}{|c|}{ Soluble solid content (Brix\%) } \\
\hline & $\mathrm{NCE}$ & FS & $\mathrm{CS}$ \\
\hline 0 & $0.4 \pm 0.1 \mathrm{a}$ & $0.4 \pm 0.1 \mathrm{a}$ & $0.4 \pm 0.1 \mathrm{a}$ \\
\hline 3 & $0.4 \pm 0.0 \mathrm{~b}$ & $0.4 \pm 0.0 \mathrm{~b}$ & $0.5 \pm 0.0 \mathrm{a}$ \\
\hline 6 & $0.4 \pm 0.0 \mathrm{a}$ & $0.4 \pm 0.0 \mathrm{a}$ & $0.4 \pm 0.0 \mathrm{a}$ \\
\hline 9 & $0.4 \pm 0.0 \mathrm{~b}$ & $0.4 \pm 0.0 \mathrm{~b}$ & $0.6 \pm 0.0 \mathrm{a}^{*}$ \\
\hline 12 & $0.4 \pm 0.0 \mathrm{~b}$ & $0.4 \pm 0.0 \mathrm{~b}$ & $0.6 \pm 0.0 \mathrm{a}^{*}$ \\
\hline \multirow[t]{3}{*}{15} & $0.4 \pm 0.0 \mathrm{a}$ & $0.4 \pm 0.0 \mathrm{a}$ & $0.4 \pm 0.0 \mathrm{a}$ \\
\hline & \multicolumn{3}{|c|}{ Hardness of cooked beans (N/seed) } \\
\hline & NCE & FS & $\mathrm{CS}$ \\
\hline 0 & $1.127 \pm 0.0 \mathrm{a}$ & $1.127 \pm 0.0 \mathrm{a}$ & $1.127 \pm 0.0 \mathrm{a}$ \\
\hline 3 & $1.188 \pm 0.1 \mathrm{a}$ & $1.111 \pm 0.0 \mathrm{a}$ & $1.262 \pm 0.1 \mathrm{a}$ \\
\hline 6 & $1.207 \pm 0.0 \mathrm{a}$ & $1.066 \pm 0.1 \mathrm{a}$ & $1.208 \pm 0.2 \mathrm{a}$ \\
\hline 9 & $1.199 \pm 0.0 \mathrm{~b}$ & $1.104 \pm 0.0 \mathrm{~b}$ & $2.267 \pm 0.0 \mathrm{a}^{*}$ \\
\hline 12 & $1.202 \pm 0.0 \mathrm{~b}$ & $1.114 \pm 0.0 \mathrm{~b}$ & $2.275 \pm 0.0 \mathrm{a}^{*}$ \\
\hline \multirow[t]{3}{*}{15} & $1.075 \pm 0.0 \mathrm{~b}$ & $1.136 \pm 0.0 \mathrm{~b}$ & $2.220 \pm 0.0 \mathrm{a}^{*}$ \\
\hline & \multicolumn{3}{|c|}{ Yield of bean paste $(\%)$} \\
\hline & NCE & FS & $\mathrm{CS}$ \\
\hline 0 & $63.53 \pm 2.8 \mathrm{a}$ & $63.53 \pm 2.8 \mathrm{a}$ & $63.53 \pm 2.8 \mathrm{a}$ \\
\hline 3 & $62.86 \pm 1.1 \mathrm{a}$ & $63.17 \pm 2.8 \mathrm{a}$ & $61.57 \pm 5.2 \mathrm{a}$ \\
\hline 6 & $61.71 \pm 4.6 \mathrm{a}$ & $62.16 \pm 4.5 \mathrm{a}$ & $61.47 \pm 4.0 \mathrm{a}$ \\
\hline 9 & $63.00 \pm 1.0 \mathrm{a}$ & $63.23 \pm 2.1 \mathrm{a}$ & $48.61 \pm 2.8 \mathrm{~b}^{*}$ \\
\hline 12 & $62.00 \pm 5.0 \mathrm{a}$ & $62.08 \pm 3.0 \mathrm{a}$ & $47.61 \pm 4.2 \mathrm{ab}^{*}$ \\
\hline 15 & $62.43 \pm 2.3 \mathrm{a}$ & $60.51 \pm 4.0 \mathrm{a}$ & $44.50 \pm 1.6 b^{*}$ \\
\hline
\end{tabular}

$\mathrm{NCE}$, natural cold energy (storage in frozen soil with a heat pipe type system ); FS, storage in a freezer at $20{ }^{\circ} \mathrm{C}$; $\mathrm{CS}$, storage at a constant temperature of $25^{\circ} \mathrm{C}$; SB, sealed bags; PB, paper bags. Data are expressed as means \pm $\mathrm{SD}(\mathrm{n}=3)$. The values with different superscript letters in the same row are significantly different among the storage conditions $(\mathrm{p}<0.01)$. The superscript $*$ indicates significant difference in the data presented in the columns compared with the values for 0 month $(\mathrm{p}<0.01)$. 

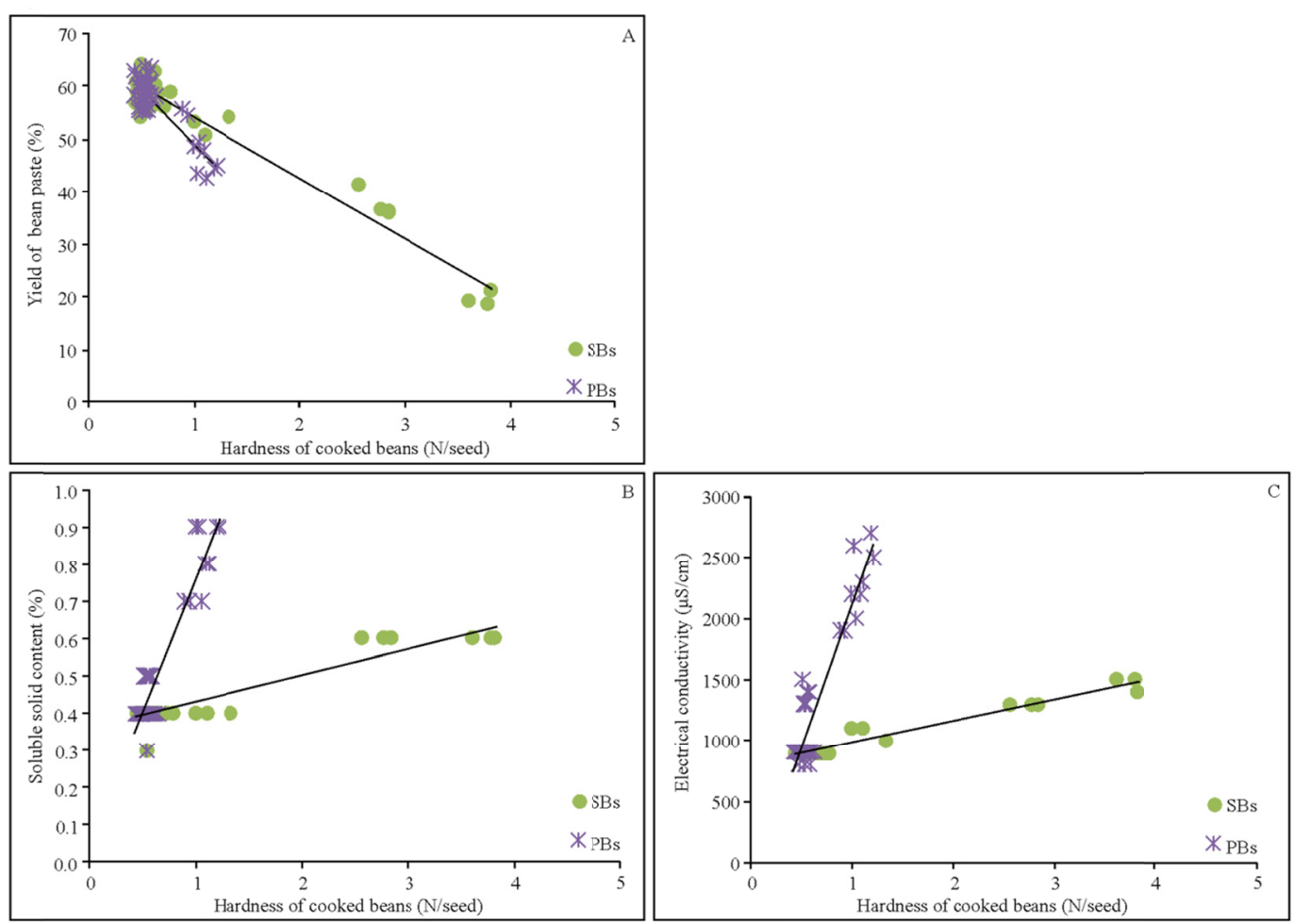

Figure 2. Correlation between yield of bean paste $(\mathrm{A}, \mathrm{rSB}=-0.970, \mathrm{rPB}=-0.861)$, soluble solid content $(\mathrm{B}, \mathrm{rSB}$ $=0.943, \mathrm{rPB}=0.944)$, electrical conductivity $(\mathrm{C}, \mathrm{rSB}=0.983, \mathrm{rPB}=0.935)$, and hardness of cooked adzuki beans stored at different storage conditions $(\mathrm{p}<0.01, \mathrm{n}=48)$


Figure 3. Correlation between yield of bean paste $(\mathrm{A}, \mathrm{rSBs}=-0.970, \mathrm{rPBs}=-0.875)$, soluble solid content $(\mathrm{B}$, $\mathrm{rSBs}=0.751, \mathrm{rPBs}=0.686)$, electrical conductivity $(\mathrm{C}, \mathrm{rSBs}=0.942, \mathrm{rPBs}=0.919)$, and hardness of cooked red kidney beans stored at different storage conditions $(\mathrm{p}<0.01, \mathrm{n}=48)$ 


\section{Discussion}

Adzuki and red kidney beans stored in SBs in frozen soil for 15 months showed good processing suitability that was similar to that obtained after storage at $-20{ }^{\circ} \mathrm{C}$. In particular, sensory evaluation of both the beans stored in SB in the frozen soil was significantly higher than that of beans stored using other methods. Therefore, a temperature of $7.1{ }^{\circ} \mathrm{C}$ and $\mathrm{RH}$ of $55.0 \%$ (SB in frozen soil storage) were considered the best storage conditions for adzuki and red kidney beans. In order to maintain a high RH and low temperature in frozen soil storage, a heat pipe is used to transfer cold energy to the underground storage location. This process does not require power and saves energy by using natural conditions to maintain a cold environment (Tsuchiya, 2004). It is likely that frozen soil storage can be efficiently implemented in Hokkaido, Japan, which has a cold climate.

The hardness of cooked beans and the amount of paste yield are important values that can predict whether the HTC phenomenon might occur. Frozen soil storage and storage at $-20{ }^{\circ} \mathrm{C}$ efficiently maintained the hardness and paste yield of both the types of beans. However, both the bean parameters increased remarkably after constant storage at $25^{\circ} \mathrm{C}$. This finding is in agreement with that of Kato et al. (2000), who showed that adzuki beans stored for extended periods at high temperatures needed a longer time to cook to attain the same tenderness as beans stored under more suitable conditions. Liu et al. (1992) also showed that long-term storage of cowpeas at $30{ }^{\circ} \mathrm{C}$ increased the seed hardness from 15.8 to $91.2 \mathrm{~N} \cdot \mathrm{g}^{-1}$, whereas there was no change in seed hardness of beans stored at $-18{ }^{\circ} \mathrm{C}$. Similarly, the hardness of common beans (Phaseolus spp.) stored at $30-35{ }^{\circ} \mathrm{C}$ increased by $3-6 \mathrm{~N}$ per seed (Del-Valle \& Stanley, 1995).

To determine the association between high temperature and HTC, Nakazato et al. (1986) investigated the effects of high temperature on kidney beans stored at high, medium, and low temperatures. They found a positive correlation between the hardness of beans and the insoluble pectin present in the cell walls and showed that beans stored at high temperature had the highest amount of insoluble pectin. On the other hand, Bhatty (1990) found that lignin content varied between beans that cooked quickly and those that took a long time to cook $(1.2-1.7 \%)$. Therefore, insoluble pectin and lignin are considered to be responsible for the development of HTC.

The processing suitability was better when the beans were placed in SB and PB in frozen soil storage, storage at $-20{ }^{\circ} \mathrm{C}$ for 15 months, or constant storage at $25^{\circ} \mathrm{C}$ for 6 months. Berrios et al. (1999) reported that black beans stored constantly at $4.5{ }^{\circ} \mathrm{C}$ with an $\mathrm{RH}$ of $50-60 \%$ exhibited the high-quality characteristics found in fresh beans, such as shorter cooking time, smaller quantities of solid loss and electrolyte leaching, and lower percentage of hardshell, than those found in beans stored under other conditions $\left(23-25^{\circ} \mathrm{C} ; \mathrm{RH}, 30-50 \%\right)$. Storage of legumes for extended periods at high temperatures $\left(30^{\circ} \mathrm{C}\right)$ increases the thickness of the cotyledon cell wall, especially of the middle lamella and secondary cell wall (Hincks \& Stanley, 1987). In our research, we found that constant humidity (SB storage) was associated with poor processing suitability of beans stored at $25^{\circ} \mathrm{C}$ (a relatively high temperature). Beans stored at this temperature also showed poor processing suitability when the storage humidity was lower. However, humidity had no effect on the processing suitability of beans stored at $-20{ }^{\circ} \mathrm{C}$ (FS) or $7.1{ }^{\circ} \mathrm{C}$ (NCE).

Electrical conductivity and soluble solid content of both types of beans showed no change after storage in frozen soil or in a $-20{ }^{\circ} \mathrm{C}$ freezer for 15 months; however, constant storage at $25{ }^{\circ} \mathrm{C}$ for 3 to 9 months significantly increased the electrical conductivity and soluble solid content. Storage of legume seeds under poor conditions (high temperature and $\mathrm{RH}$ ) for extended periods damages their cell membrane, allowing divalent cations to leak from the cells (Liu, 1995).

The SMC was retained by storing the adzuki beans in SB. Similarly, features associated with high quality (percentage weight loss, moisture content, fatty acid content, and color) of corn grains stored in sealed enclosures were well maintained with minimal damage by insects at the completion of the storage trial (Gras et al., 1990).

Bean hardness had a high positive correlation and paste yield had a negative correlation with electrical conductivity and soluble solid content of the soaking liquid of adzuki and red kidney beans. This finding suggests that the electrical conductivity and soluble solid content of the liquid used to soak beans may be used to evaluate the processing suitability of cooked beans and the paste yield of adzuki and red kidney beans. Nasar-Abbas et al. (2008) reported that solute and electrolyte leakage and the hardness of faba beans stored at $5-50{ }^{\circ} \mathrm{C}$ increased with increasing storage temperature. The soluble solid content of soaking liquid of soybeans stored under different storage conditions was correlated with processing suitability (Hira, 1983). Therefore, some researchers have hypothesized that electrical conductivity and the release of soluble solid content from bean cells might reduce their water affinity and water-holding capacity in accordance with osmotic principles, thereby affecting the processing suitability of beans (Jones \& Boulter, 1983; Berrios et al., 1999; Nasar-Abbas et al., 
2008).

\section{Conclusions}

The results of this study indicated that the deterioration of processing suitability of adzuki and red kidney beans could be inhibited by using frozen soil storage. When both types of beans were stored in sealed bags in a natural cold storage system, their taste was retained and the HTC phenomenon was prevented. Electrical conductivity value and soluble solid content of the liquid used for soaking beans may be used to evaluate the processing suitability of cooked beans.

\section{Acknowledgments}

We wish to sincerely thank Prof. F. Tsuchiya at the Obihiro University of Agriculture and Veterinary Medicine, for kindly providing the storage facility, and Prof. H. Koaze at the same university for his valuable advice regarding the texture analysis experiment. We also acknowledge the financial support provided by Obihiro University of Agriculture and Veterinary Medicine for the completion of the study.

\section{References}

Berrios, J. D. J., Swanson, B. G., \& Cheong, W. A. (1999). Physico-chemical characterization of stored black beans (Phaseolus vulgaris L.). Food Research. International, 32, 669-676. http://dx.doi.org/10.1016/S0963-9969(99)00144-1h

Bhatty, R. S. (1990). Cooking quality of lentils: The role of structure and composition of cell walls. Journal of Agricultural and Food Chemistry, 38, 376-383. http://dx.doi.org/10.1021/jf00092a007

Cunniff, P. (1995). AOAC, Official Methods of Analysis of AOAC International (16th ed.), Arlington, VA: AOAC International.

Del-Valle, J. M., \& Stanley, D. W. (1995). Reversible and irreversible components of bean hardening. Food Research International, 28, 455-463.

El-Refai, A. A., Harras, H. M., El-Nemr, K. M., \& Noaman, M. A. (1988). Chemical and technological studies on faba bean seeds. I-Effect of storage on some physical and chemical properties. Food Chemistry, 29, 27-39. http://dx.doi.org/10.1016/0308-8146(88)90073-8

Feng, S., Wu, S., \& Kojima, M. (2015). Effect of storage in frozen soil on the quality of adzuki beans, soybean, red kidney beans, and buckwheat. Submitted to Journal of Food Nutrition Research.

Giami, S. Y., \& Okwechime, U. I. (1993). Physicochemical properties and cooking quality of four new cultivars of Nigerian cowpea (Vigna unguiculata L Walp). Journal of the Science of Food and Agricukture, 63, 281-286. http://dx.doi.org/10.1002/jsfa.2740630304

Gras, P. W., Bason, M. L., Esteves, L. A., Sabio, G. C., Annis, P. C., \& Graver, J. E. van S. (1990). Quality changes in maize stored in sealed bag stacks. Journal of Stored Product Research, 26, 199-206. http://dx.doi.org/10.1016/0022-474X(90)90022-K

Hatai, A. (1994). Cooking characters of adzuki beans. Science of Cookery, 27, 238-242. (in Japanese).

Hincks, M. J., \& Stanley, D. W. (1987). Lignification: Evidence for a role in hard-to-cook beans. Journal of Food Biochemistry, 11, 41-58. http://dx.doi.org/10.1111/j.1745-4514.1987.tb00111.x

Hira, H. (1983). Quality of domestic soybean -3- correlation of processing suitability, physical properties and chemical composition. Report of National Food Research Institute, 42, 27-39. (in Japanese).

Japan Meteorological Agency (2015). Temperature and humidity of each year in Obihiro City, Hokkaido, Japan. Retrieved http://www.data.jma.go.jp/obd/stats/etrn/view/annually_s.php?prec_no $=20 \& b l o c k \_n o=47417 \&$ year $=2010 \&$ month $=\&$ day $=\&$ view $=$ p1 (in Japanese).

Jones, P. M. B., \& Boulter, D. (1983). The cause of reduced cooking rate in Phaseolus vulgaris following adverse $\begin{array}{lllll}\text { storage conditions. Journal of Food Scieince, } & \text { 48, }\end{array}$ http://dx.doi.org/10.1111/j.1365-2621.1983.tb10804.x

Kagawa, Y. (2010). Standard tables of food composition in Japan - Fifth Revised and Enlarged Edition. Tokyo, Japan: Kagawa Nutrition University Publications Division. (in Japanese).

Kato, J. (2002). Estimation method for characteristics for boiling of beans by using electric conductivity of soaking water. Journal of Science of Soil and Manure, Japan, 73, 181-184 (in Japanese). 
Kato, J., Yousif, A. M., Deeth, H. C., Suzuki, M. M., Caffin, N. A., Meguro, T. (2000). Differences in the cooking quality between two adzuki varieties harvested in Australia and stored at different temperatures. Journal of Cookery Science of Japan, 33, 257-266.

Kokubun, M. (2010). New revised food crops. Tokyo, Japan:Yokendo Co. (in Japanese).

Liu, K. (1995) Cellular, biological, and physicochemical basis for the hard-to-cook defect in legume seeds. Critical Review of Food Scieince and Nutriton, 35, 263-298. http://dx.doi.org/10.1080/10408399509527702

Liu, K., McWatters, K. H., \& Phillips, R. D. (1992). Protein insolubilization and thermal destabilization during storage as related to hard-to-cook defect in cowpeas. Journal of Agriculture and Food Chemistry, 40, 2483-2487. http://dx.doi.org/10.1021/jf00024a028

Ministry of Agriculture, Forestry and Fisheries. (2010). Yield of beans (dry grain) and buckwheat. Statistics Bureau, Ministry of Internal Affairs and Communications. Retrieved from http://www.e-stat.go.jp/SG1/estat/List.do?lid=000001081820 (in Japanese).

Moscoso, W., Bourne, M. C., \& Hood, L. F. (1984). Relationship between the hard-to-cook phenomenon in red kidney beans and water absorption, puncture force, pectin, phytic acid and minerals. Journal of Food Science, 49, 1577-1583. http://dx.doi.org/10.1111/j.1365-2621.1984.tb12848.x

Nakazato, T., Ichikawa, T., Mitani, T., \& Sasaki, I. (1986). Variation of kidney beans quality during storage: changes in pectin, calcium and magnesium. Science of Cookery, 19, 281-284 (in Japanese).

Nasar-Abbas, S. M., Plummer, J. A., Siddique, K. H. M., White, P., Harris, D., \& Dods, K. (2008). Cooking quality of faba bean after storage at high temperature and the role of lignins and other phenolics in bean hardening. LWT-Food Science Technology, 41, 1260-1267. http://dx.doi.org/10.1016/j.lwt.2007.07.017

Planning and Follow-up Group, Development Research Division, Development Administration (2010). Survey on transport that utilize the snow and ice cold of the cold storage of agricultural products. Ministry of Land, Infrastructure, Transport and Tourism Hokkaido Regional Development. Retrieved June 2010, from http://www.hkd.mlit.go.jp/topics/toukei/chousa/h22keikaku/03.pdf (in Japanese).

Reyes-Moreno, C., Paredes-López, O., \& Barradas, I. (1994). A fast laboratory procedure to assess the hard-to-cook tendency of common bean varieties. Food Chemistry, 49, 187-190. http://dx.doi.org/10.1016/0308-8146(94)90157-0

Tsuchiya, F. (2004). Artificial permafrost storage using heat pipes. Journal of Japan Society of Snow and Ice, 66, 251-257 (in Japanese).

Tsuchiya, F., Ishibashi, K., Ryokai, K., \& Yoshida, H (1994). Studies on effects of the system on artificial permafrost facilities using heat pipes and abilities of storage for vegetables and rice. Proceedings of Cold Region Technology Conference, 10, 619-626 (in Japanese).

Tsuchiya, F., \& Ryokai, K. (1990). Low temperature storage room of artificial permafrost using heat pipes. Journal of JSIDRE, 58, 881-886 (in Japanese).

Tsuchiya, F., \& Ryokai, K. (1996). Cold storage system surrounded by frozen soil using cold energy. Journal of JSIDRE, 64, 231-235 (in Japanese).

Watanabe, T. (2000) Encyclopedia of beans: processing and utilization. Tokyo, Japan: Saiwai Shobo Co (in Japanese) .

Yousif, A. M., Deeth, H. C., Caffin, N. A., \& Lisle, A. T. (2002). Effect of storage time and conditions on the hardness and cooking quality of adzuki (Vigna angularis). LWT-Food Science Technology, 35, 338-343. http://dx.doi.org/10.1006/fstl.2001.087

\section{Copyrights}

Copyright for this article is retained by the author(s), with first publication rights granted to the journal.

This is an open-access article distributed under the terms and conditions of the Creative Commons Attribution license (http://creativecommons.org/licenses/by/3.0/). 